\title{
CROWD CONTROL SYSTEM USING IR TRANSMITTER AND RECEIVER
}

\author{
Yashashree Shelke $^{1}$, Vrushali Patil ${ }^{2}$, Sayali Desale ${ }^{3}$, R.S. Jagale ${ }^{4}$ \\ ${ }^{I}$ Nasik, Department of Computer Engineering, GES RHSCOE, Nasik, India \\ ${ }^{2}$ Nasik, Department of Computer Engineering, GES RHSCOE, Nasik, India \\ ${ }^{3}$ Nasik, Department of Computer Engineering, GES RHSCOE, Nasik, India \\ ${ }^{4}$ Nasik, Department of Computer Engineering, GES RHSCOE, Nasik, India
}

\begin{abstract}
An efficient crowd control system is needed for safety of lives, property, time and economy. Crowd control system presents a design and implementation of low cost, low power consummated and more reliable and an infrared based intelligent crowd control system. The system contains Infrared transmitters and receiver. The basic concept of IR (Infrared) obstacle detection is to transmit the IR signal (radiation) in a direction and signal is received at the IR receiver when the IR radiation bounces back from a surface of the object. The system can response rapidly with violation of crowd limit. System describes highly accurate crowd control system using infrared communication. Proposed system achieves high accuracy and more efficiency at four way terminals. In every direction the road will consist of an IR transmitter-receiver pair at a certain distance. When crowd will be heavy in one particular direction during emergency situation it will indicate the administrator by sending message. So the heavy crowd can be routed to other route by preventing the stampede.
\end{abstract}

Keywords: Crowd, IR transmitter, IR receiver, Stampede

\section{INTRODUCTION}

Now a day's Human crowd on the roads is the burning issue. To control this exploding crowd we need a dynamic system. Here, we are proposing the system which deals with the same problem and the system is CROWD CONTROL SYSTEM USING IR TRANSMITTER AND RECEIVER. This system is based on the principle of IR transmitter and receiver sensors. Proposed dynamic system which will alert about the heavy crowd gathered in a particular region. This system will be mounted on every road and each junction which are located in the area where people can gather. For each system a local administrator will be provided and to control and connect these various local administrator. A central administrator will be allocated. Presence of human at mounted system will inform the local administrator and if a heavy crowd is present on road then the alert message will be send to the central administrator by the local administer, after crowd analysis. The central administrator will take decisions and send the decision to the volunteers hence the crowd can be control preventing the mishap. The main aim and objective of the crowd control system are:

- By using IR transmitter and receiver detect the human presence.

- To track down the approximate human density at various points in the city.

- To develop a dynamic platform for current human traffic update.

\section{RELATED WORK}

The main element of crowd control system is IR transmitter receiver pair. IR LED emits infra-red radiation. IR LED is used as a source of infrared rays. It comes in two packages $3 \mathrm{~mm}$ or $5 \mathrm{~mm} .3 \mathrm{~mm}$ is better as it is requires less space.[2] IR sensor is nothing but a diode, which is sensitive for infrared radiation. This infrared transmitter and receiver is called as IR TX-RX pair. Color of IR transmitter and receiver is different. Features:

1. High Reliability

2. High Radiant Intensity

3. peak wavelength

4. Low Forward Voltage

The emission is carried out in a continuous way by the emitters; and when the receivers do not detect this emission, the presence of an obstacle can be concluded.[3]

\section{PROJECT SCOPE}

From years, we are using CCTV cameras for such crowdy situations for monitoring. But in case of CCTV cameras they require light to catch the scenario i.e. the CCTV cameras are not useful during night when the electric power supply is not available unfortunately. Hence the system with CCTV cameras fails to capture the pictures in such conditions stated above. Five related concepts are encompassed by the general scope of the Crowd Control System. 
1] The first pertains to the replacement of CCTV cameras by hardware model mounted with IR transmitters and receivers.

2] The second is decreasing the importance of man power and make maximum use of the system software. In this case, analysis of input data, conclusion from it and decision making is done by system.

3] The third concept is, the decision of the system is send via SMS to the central system and this system sends further SMS to the related locations for crowd controlling.

4] The fourth concept relates with the memory require for the database. As the output of the system is only in the form of numbers so it requires less memory compared to the present system.

5] This system provides a user friendly platform containing a horizontal bar graph which is easy to understand current crowd status. It is not necessary that users must have a smart phone to access this web page.

\section{CROWD CONTROL SYSTEM}

There are three main sections to this system, the human presence detection, crowd analysis and crowd routing. For human presence detection, the IR transmitter-receiver pairs are used where transmitter produces $38 \mathrm{KHz}$ carrier wave. The maximum distance between a transmitter and a receiver is up to 300 meter, but in such a long distance the transmitter signal weakens so for that frequency modulation technique is used.

\subsection{IR Transmitter Receiver Pair Model with}

\section{Transistor}

Here we have designed a hardware model which consists of IR Transmitter-Receiver Pairs, Transistors, LED's and various Registers. Transmitted rays from the transmitter are received by the receiver as there is no obstacle present between the pair. Following is the circuit diagram of proposed system using all the hardware mentioned above.

Here we have designed a circuit using IR transmitter and receiver. A $220 \mathrm{ohm}$ resistor is connected in series with transmitter where transmitter is forward biased and this end is grounded. The receiver is reverse biased connected in series with a $10 \mathrm{k} \mathrm{ohm}$ resistor. For this connection further we have connected a $1 \mathrm{k} \mathrm{ohm}$ resistor which is again in series with transistor BC547. Base of BC547 is in series with $1 \mathrm{k} \mathrm{ohm}$ resistors ,emitter is grounded and collector is connected in series with a small circuit of LED and a resistor of $1 \mathrm{k} \mathrm{ohm}$.

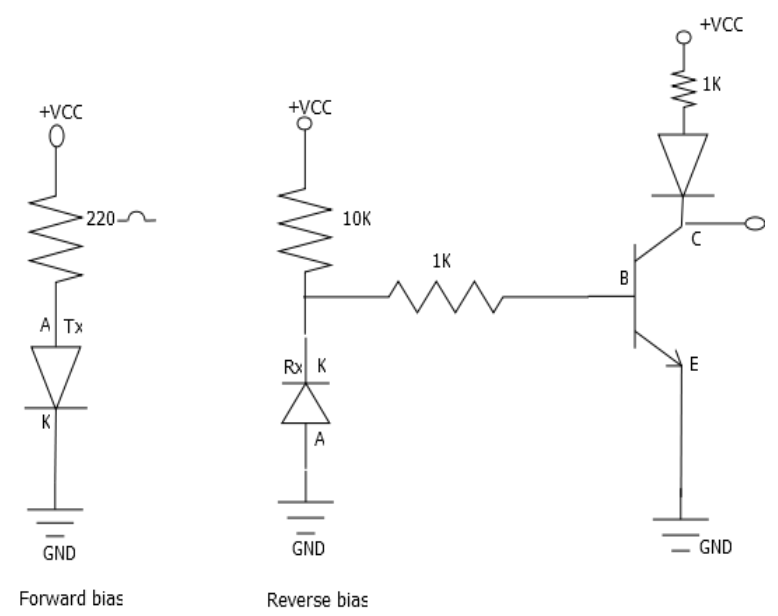

Fig -1: Circuit Diagram of hardware made

The distance between two transmitters - receiver pair is not fixed. We can change it according to our requirement. For this case, the transmitter- receiver pairs are separated by $1 \mathrm{ft}$. The distance is $1 \mathrm{ft}$. because a person occupies near about $1 \mathrm{sq}$. $\mathrm{ft}$. space to stand in a queue. So at a time a single transmitter receiver pair can detect presence of a person standing in between. Hence $1 \mathrm{ft}$ distance between the pairs can approximately measure the crowd present on the road.

The output generated by above hardware model is sent to the software module for further processing.

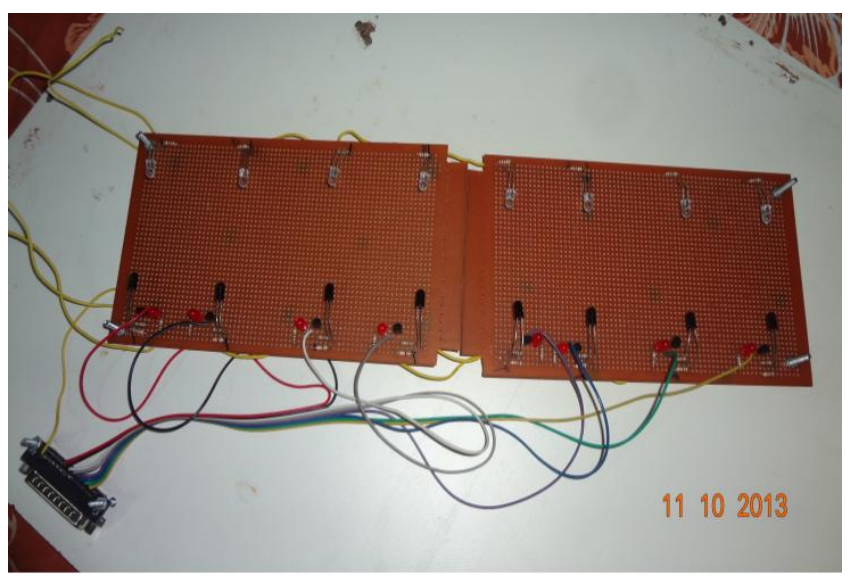

Fig -2: Hardware model

\subsection{Software Module:}

\subsubsection{Login (Administrator) :}

We have designed this web page for the authorized person i.e. administrator. The past information about the crowd, stored in the database can be accessed by this administrator whenever he wants. Here we have provided a user id and password for the administrator. 


\subsubsection{Monitoring Panel}

The monitoring panel is designed for the Central Administrator. This panel is used for the monitoring of current status of all booths installed for crowd control system. This page must require the username and password to access it.

\subsubsection{Graphical Display}

On a webpage designed, we will have shown the graphical display which will give the current information about the crowd status in a particular area. This graphical display is in a form of horizontal bar graph. When the user wants to know about the crowd status in a particular area, he just has to visit the webpage having the graphical display which will give the idea about the crowd.

\subsubsection{SMS Firing Module}

When the crowd is beyond the limit then this SMS firing module will be activated and it will send a SMS to the authority about the crowd status and crowd location.

\section{SYSTEM ARCHITECTURE}

The figure 3 shows the whole architecture of the proposed system. When human makes an interrupt between IR transmitter receiver pair model ,the presence of obstacle will be determined by IR pair. Then that input from the IR pair will be given to transistor logic which will then generates the output in the form of binary digit number. Then this output from the hardware model will be given to system that is local administrator which also act as a server. Then local administrator will analyses the crowd status and generates the two kind of outputs. If crowd will violating the limit then local administrator will send the alert message to central administrator for further decision making. System also gives the output in terms of web page which will show the current status of a crowd in terms of horizontal bar graph. System also update the web page time to time. After receiving the message from local administrator, central administrator will take a decision according to the situation and send that decision to volunteer. Then volunteer will follow that decision and controls the crowd. Central administrator also watch the monitoring panel to get the updates related to crowd and updates database time to time. Remote user can able to access the web page for current information about the status of the crowd. Remote user get the status in terms of graphical display. The web page can be accessible by central administrator, local administrator, volunteer also.

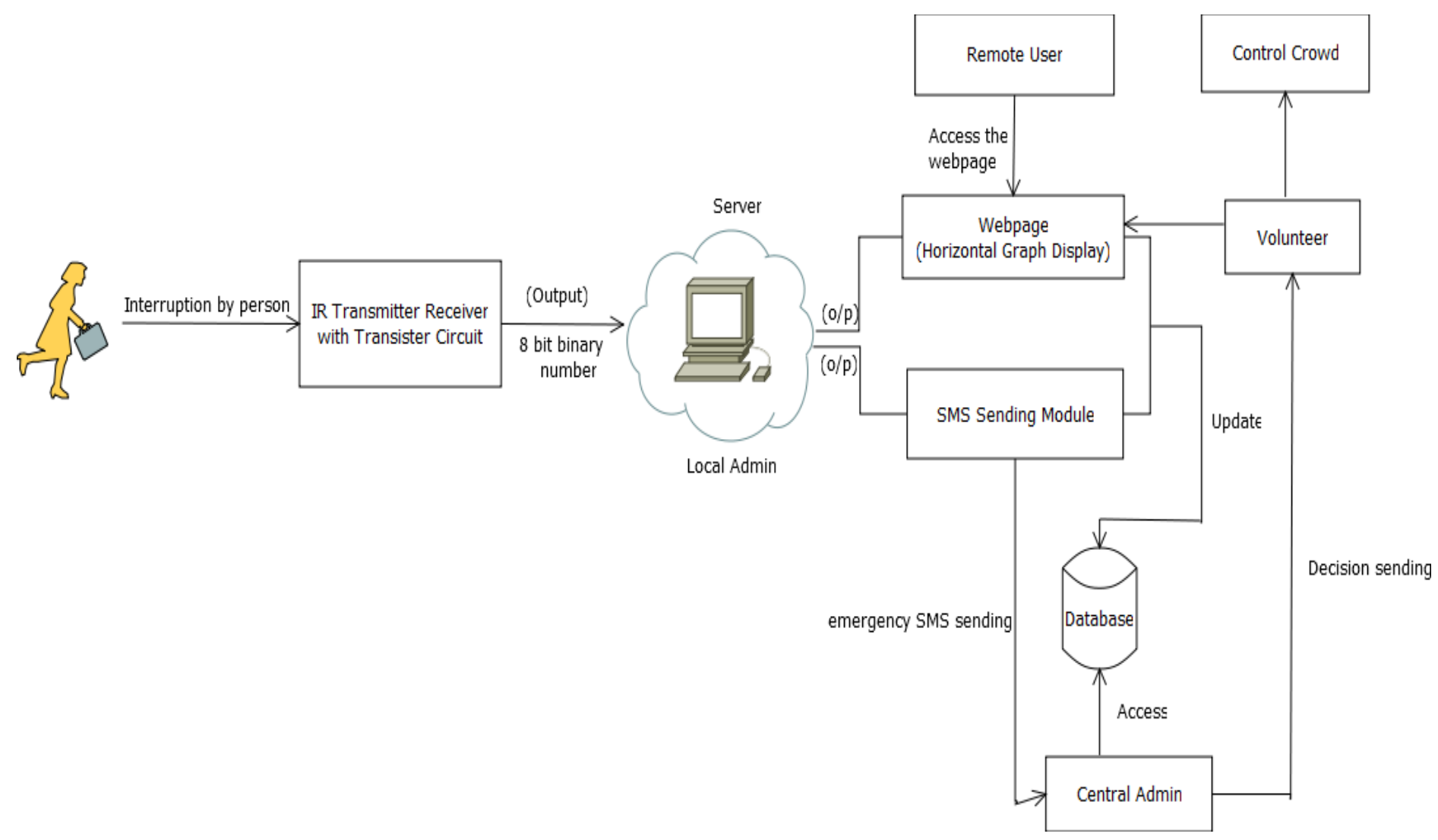

Fig -3: System Architecture of the System 


\subsection{Applications}

This system is useful in crowd control on road, at four way junctions. The system mounted on the road pavements will be useful in on road crowd control. The crowd at the four way junctions will also be controlled by simple modification in design of the same system.

- On road traffic will be controlled by this system.

- This system is also useful at the restricted areas like jewelers shops, banks, godam etc.

- This system can be mounted on the entrance and exit of the stadium to control the crowd.

\subsection{Advantages}

This system is cheaper than the present system. It is less expensive option for crowd control. No need of power supply for object detection. The IR transmitter receiver does not require any extra light for obstacle detection. Crowd status of each moment is known. The system is totally dependent on the internet connection. so, the crowd status of each and every second is known to the authority and to the end users. Accessible on the simple phone. A simple phone containing the GPRS system also can access the information on the dedicated web page. Proposed system is very easy to use. As the user just need to visit the web page specially created for the users. The crowd information is available for the user on a single click.

\subsection{Results}

First the interruptions is made by person on the road an detected by hardware model i.e. through IR transmitter and receiver. This output of hardware model goes to our system as a input $n$ we will get our expected results. A remote user can access our main webpage when he wants to see the current crowd status of respective areas.

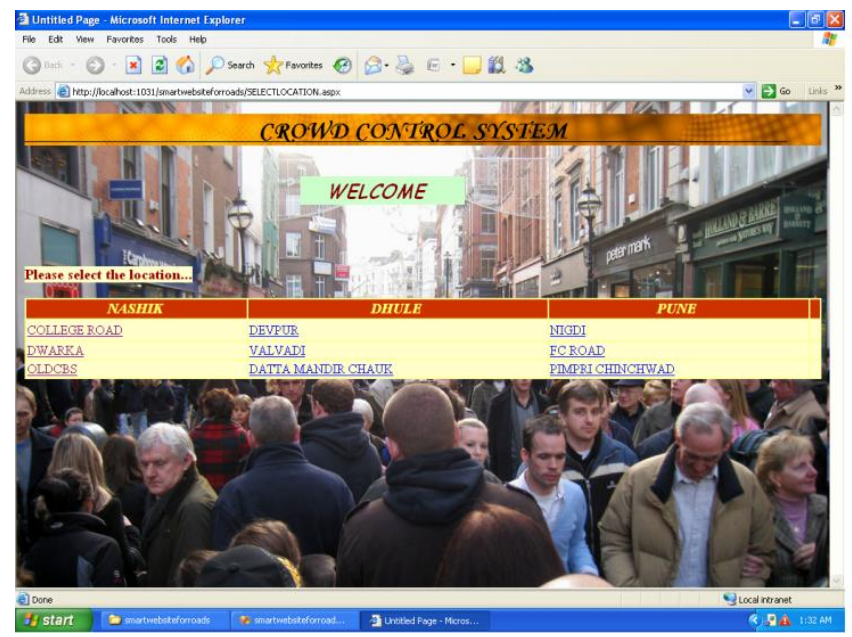

Fig -4: Select location page
This is select location page from which user will select the area for knowing the current crowd status. This is the webpage for remote user or any people who wanted to know the current crowd status.

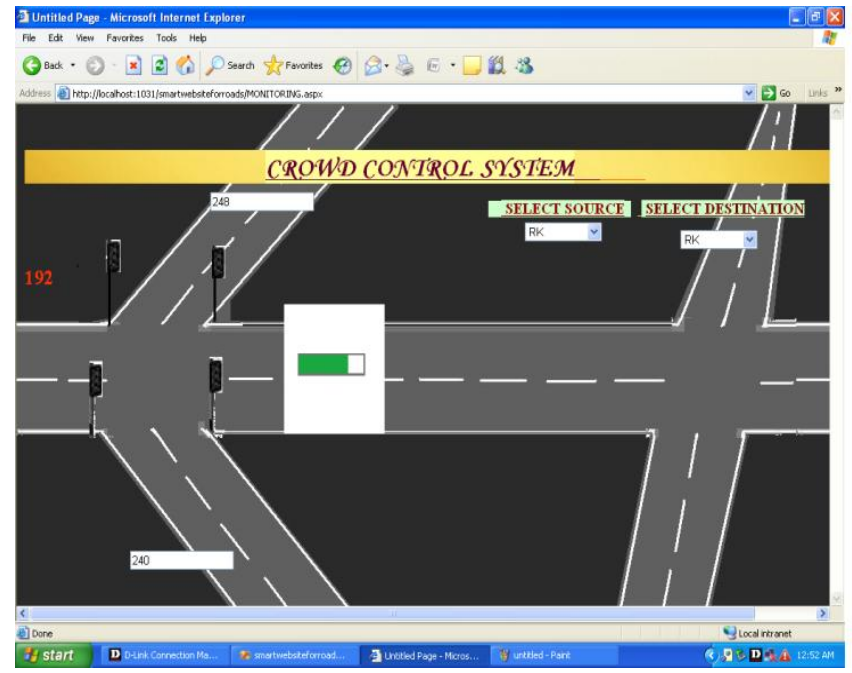

Fig -5: Monitoring page 1

After selecting the location from the select location page user will see the monitoring page as shown in fig 5. On the monitoring page user can see the current crowd status in the form bar graph in green color.

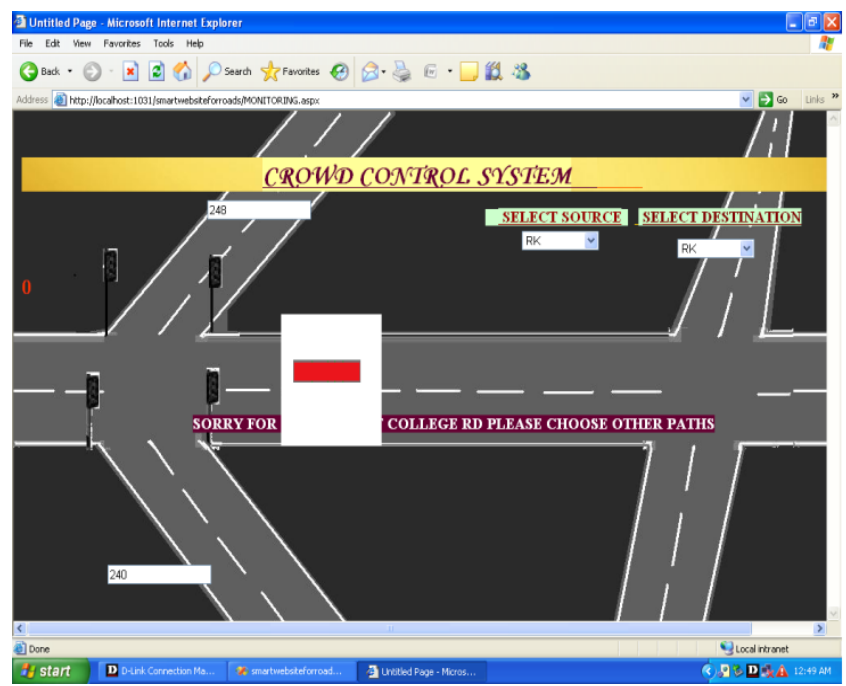

Fig -6: Monitoring page 1

Whenever road is full of crowd the bar graph is in red color and we will have the message blinking on webpage "SORRY FOR ROAD JAM SO PLEASE CHOOSE OTHER PATHS" as shown in fig 6 and the movement road is jam the higher authority i.e. recipients no will get a information message about road jam. 


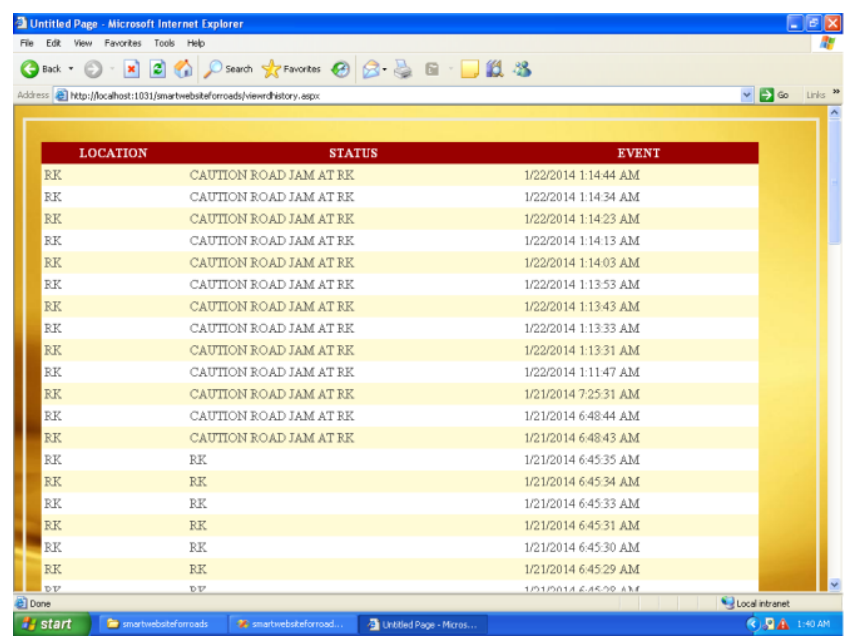

Fig -7: History page

We also maintain the database when there is jam at road. We provided the username and password to the authority. Only a authorized person can see this database history so that admin can take the decisions for routing the people.

\section{CONCLUSIONS}

Human traffic i.e. crowd is routed through the right path using the IR Transmitter and Receiver pairs. This is very efficient and durable way to control the crowd. Flexibility is provided within cheaper cost. No need to maintain a huge database. Instant action is taken for alert messages sent. Hence, it is feasible to use the proposed system for crowd controlling.

\section{REFERENCES}

[1] Dereniak E.L. and Boreman, G.D. (1996) "Infrared Detectors and Systems." New York: John Wiley.

[2] IEEE/OSA/IAPR International Conference on Infonnatics, Electronics \& Vision,"Infrared based traffic intelligent system" May 2011

[3] IEEE base paper on," Dedicated smart IR barrier for obstacle detection in railways", May 2005

[4] Technical details of IR transmitter and receiver available @ http://electrosome.com/ir-transmitterreceiver-led-tsop1738/

\section{BIOGRAPHIES}

First Author - Miss. Yashashree shelke, Department of Computer Engineering, GES's RHSCOE, yashashree.shelke7@gmail.com

Second Author - Miss. Vrushali Patil, Department of Computer Engineering, GES's RHSCOE, patilvrushali146@gmail.com
Third Author - Miss. Sayali Desale, Department of Computer Engineering, GES's RHSCOE, sayali.deasle27@gmail.com.

Fourth Author - Mr. R. S. Jagale, Department of Computer Engineering, GES's RHSCOE, rjagale@gmail.com. 